\title{
Regularity of the Donaldson Geometric Flow
}

\author{
Robin S. Krom ${ }^{1}$
}

Received: 25 January 2020 / Revised: 7 June 2021 / Accepted: 20 June 2021 /

Published online: 2 October 2021

(C) The Author(s) 2021

\section{Abstract}

We prove a regularity theorem for the solutions of the Donaldson geometric flow equation on the space of symplectic forms on a closed smooth four-manifold, representing a fixed cohomology class. The minimal initial conditions lay in the Besov space $B_{2}^{1, p}\left(M, \Lambda^{2}\right)$ for $p>4$. The Donaldson geometric flow was introduced by Simon Donaldson in Donaldson (Asian J. Math. 3, 1-16 1999). For a detailed exposition see Krom and Salamon (J. Symplectic Geom. 17, 381-417 2019).

Keywords Donaldson geometric flow · Uniqueness of symplectic structures

Mathematics Subject Classification (2010) $35 \mathrm{Kxx} \cdot 58 \mathrm{~J} 35 \cdot 58 \mathrm{~J} 90$

\section{Introduction}

Let $M$ be a smooth closed Riemannian four-manifold. Denote by $g$ the Riemannian metric, denote by dvol $\in \Omega^{4}(M)$ the volume form of $g$ and let $*: \Omega^{k}(M) \rightarrow \Omega^{n-k}(M)$ be the Hodge $*$-operator associated to the metric and orientation. Let $\omega$ be a symplectic form on $M$ compatible with the metric and let $\mathscr{S}_{a}$ be the space of symplectic forms representing the cohomology class $a=[w] \in H^{2}(M ; \mathbb{R})$. This is formally an infinite dimensional manifold and the tangent space at any element $\rho \in \mathscr{S}_{a}$ is the space of exact two-forms. The Donaldson geometric flow on $\mathscr{S}_{a}$ is given by the evolution equation

$$
\frac{d}{d t} \rho_{t}=d *^{\rho_{t}} d \Theta^{\rho_{t}}
$$

where $\Theta^{\rho}$ and $*^{\rho}$ are defined pointwise on $M$ by the equations

$$
\Theta^{\rho}:=* \frac{\rho}{u}-\frac{1}{2}\left|\frac{\rho}{u}\right|^{2} \rho, \quad \frac{1}{2} \rho \wedge \rho=: u \text { dvol, } \quad *^{\rho} \lambda:=\frac{\rho \wedge *(\rho \wedge \lambda)}{u}
$$

Robin S. Krom

drsk@lavabit.com

Zürich, Switzerland 
for all one-forms $\lambda \in \Omega^{1}(M)$. The operator $*^{\rho}$ is the Hodge star operator for the metric $g^{\rho}$ which is uniquely determined by the conditions

$$
\operatorname{dvol}_{g} \rho=\mathrm{dvol}, \quad *^{\rho}\left(\omega-\frac{\omega \wedge \rho}{\operatorname{dvol}_{\rho} \rho}\right)=\left(\omega-\frac{\omega \wedge \rho}{\operatorname{dvol}_{\rho} \rho}\right) \Leftrightarrow * \omega=\omega
$$

for all $\omega \in \Lambda^{2} T^{*} M$. A detailed explanation of the construction of the metric $g^{\rho}$ and the operator $*^{\rho}$ is given in [5]. Each $\rho \in \Omega^{2}$ with $\rho^{2}>0$ determines an inner product $\langle\cdot, \cdot\rangle_{\rho}$ on the space of exact two-forms defined by

$$
\left\langle\widehat{\rho}_{1}, \widehat{\rho}_{2}\right\rangle_{\rho}:=\int_{M} \lambda_{1} \wedge *^{\rho} \lambda_{2}, \quad d \lambda_{i}=\widehat{\rho}_{i}, \quad *^{\rho} \lambda_{i} \text { is exact. }
$$

These inner products determine a metric on the infinite dimensional space $\mathscr{S}_{a}$ called the Donaldson metric. The Donaldson geometric flow is the negative gradient flow with respect to the Donaldson metric of the energy functional $\mathscr{E}: \mathscr{S}_{a} \rightarrow \mathbb{R}$ defined by

$$
\mathscr{E}(\rho):=\int_{M} \frac{2\left|\rho^{+}\right|^{2}}{\left|\rho^{+}\right|^{2}-\left|\rho^{-}\right|^{2}}, \quad \rho \in \mathscr{S}_{a} .
$$

The Donaldson flow equation has a beautiful geometric origin laid out in Donaldson's paper [1]. The key idea is that the space of diffeomorphisms of a hyperKähler surface has the structure of an infinite dimensional hyperKähler manifold. The group of symplectomorphisms with respect to a preferred symplectic structure $\omega$ then acts by composition on the right and this group action is generated by a hyperKähler moment map. In analogy with the finite dimensional case, one then studies the negative gradient flow to the moment map square functional with respect to the $L^{2}$-inner product. If we push the preferred symplectic structure $\omega$ forward by the diffeomorpisms of $M$ to the space of symplectic structures in a fixed cohomology class, we obtain the Donaldson flow equation (1). If we push the moment map square forward, we obtain the energy functional $\mathscr{E}: \mathscr{S}_{a} \rightarrow \mathbb{R}$ in (3) and if we push the $L^{2}$-metric forward, we obtain the Donaldson metric (2). The Donaldson flow remains the negative gradient flow to this energy functional with respect to the Donaldson metric (2) on the space of symplectic structures in a fixed cohomology class. The Donaldson flow remains well defined for a general symplectic 4-manifold $(M, \omega)$ equipped with a compatible Riemannian metric $g$.

The motivation to study the Donaldson flow on the space of symplectic structures comes from the longstanding open uniqueness problem for symplectic structures on closed fourmanifolds (see [9] for an exposition). The hope is that the Donaldson flow provides a tool to settle this question at least in some favorable cases, such as the hyperKähler surface and the complex projective plane $\mathbb{C P}^{2}$. This hope is strengthened by the observation that the preferred symplectic structure $\omega$ is the unique absolute minimum and that the Hessian of the energy functional $\mathscr{E}$ is positive definite at the absolute minimum (see [5]). In the case of $M=\mathbb{C P}^{2}$, we can further show that the Fubini-Study form is the only critical point of the flow. Thus, if longtime existence and convergence of the Donaldson flow can be established, the Donaldson flow would provide a proof for the uniqueness of the symplectic structures on $\mathbb{C P}^{2}$ of a given cohomology class up to isotopy. In the case of the hyperKähler surface, Donaldson [1] proved that the higher critical points are not strictly stable. The idea would then be to perturb the flow near the critical points such that eventually the perturbed flow converges towards the unique absolute mimimum.

The main result of this paper is the regularity for critical points and the regularity for the flow (Section 4). Local existence and the semiflow property of the Donaldson flow have been established in the Ph.D. thesis of the author [6]. These results lay the foundation 
for future studies focusing on longtime existence and the problem of solutions escaping to infinity. Section 2 contains the main geometric ideas, in particular a result by Donaldson [2] that shows that the map $\rho \mapsto \frac{\rho^{+}}{u}$ from the space of symplectic forms representing a cohomology class in a fixed affine space in $H^{2}(M ; \mathbb{R})$ with dimension equal to $b_{2}^{+}$to the space of self-dual two-forms is a local Banach space diffeomorphism. In Section 3 we then study the evolution of $\frac{\rho^{+}}{u}$ if $\rho$ evolves by the Donaldson flow equation. This evolution equation is the key to prove the regularity theorem for solutions of the Donaldson flow equation in Section 4.

Notation and Conventions Let $(M, g)$ be a closed Riemannian oriented four-manifold. Let $\pi: E \rightarrow M$ be a natural $k$-dimensional vector bundle over $M$. We denote by $W^{\ell, p}(M, E)$ the Sobolev completion of the space of sections $\Omega^{0}(M, E)$. If the bundle in question is clear from the context, we will just write $W^{\ell, p}$ instead of $W^{\ell, p}(M, E)$. We will suppress the constants that solely depend on the parameters $\operatorname{dim}(M), \operatorname{vol}(M), k, p$ from the notations when we make estimates in Sobolev norms. We denote by $\mathscr{S}$ the space of smooth symplectic structures on $M$ compatible with the orientation. We write $\mathscr{S}_{a}$ for $\rho \in \mathscr{S}$ that represent a fixed cohomology class $a \in H^{2}(M ; \mathbb{R})$. Let $L \in H^{2}(M ; \mathbb{R})$ be an affine subspace. Then, $\mathscr{S}_{L}$ denotes the subset of $\mathscr{S}$ such that the symplectic structures represent cohomology classes in $L$. We define

$$
\mathscr{S}^{k, p}:=\left\{\rho \in W^{k, p}\left(M, \Lambda^{2}\right) \mid \rho \wedge \rho>0, d \rho=0\right\} .
$$

A cohomology class or an affine subspace of cohomology classes as subscript has the same meaning as in the smooth counterpart. A linear subspace $\mathcal{H} \subset H^{2}(M ; \mathbb{R})$ is called positive, if and only if for all $0 \neq a \in \mathcal{H}$ we have $a^{2}([M])>0$, where $[M]$ denotes the fundamental homology class of $M$. Finally, a polynomial with positive real coefficients in several variables is a polynomial given by

$$
\mathfrak{p}\left(x_{1}, \ldots, x_{\ell}\right)=\sum_{|\alpha| \leq m} a_{\alpha} x^{\alpha},
$$

where the sum runs over all multi-indices $\alpha=\left(\alpha_{1}, \ldots, \alpha_{\ell}\right) \in \mathbb{N}_{0}^{\ell}$ with $|\alpha|=\sum_{i=1}^{\ell} \alpha_{i} \leq m$ and $a_{\alpha} \geq 0$ for all $\alpha$.

\section{The Map $K$}

In this section, we study the map

$$
\mathscr{S} \rightarrow \Omega^{+}: \quad \rho \rightarrow \frac{\rho^{+}}{u}, \quad u:=\frac{\rho \wedge \rho}{2 \mathrm{dvol}}
$$

from the space of symplectic forms to the space of self-dual two-forms. We apply a theory developed by Donaldson [2] on elliptic problems for closed two-forms on four dimensional closed manifolds that fullfill a pointwise constraint with 'negative tangents'. The main insight is that this map is a local Banach space diffeomorphism between appropriately choosen spaces and the regularity of $\frac{\rho^{+}}{u}$ determines the regularity of $\rho$. The precise statement is given in Theorem 1.

We will use the construction of a Riemannian metric determined by a nondegenerate two-form and a volume form explained in [5]. Here is a brief overview. Fix a nondegenerate two-form $\rho \in \Omega^{2}(M)$ such that $\rho \wedge \rho>0$. There exists a Riemannian metric $g^{\rho}$ such 
that its volume form agrees with dvol of $(M, g)$. The associated Hodge star operator $*^{\rho}$ : $\Omega^{1}(M) \rightarrow \Omega^{3}(M)$ is given by

$$
*^{\rho} \lambda=\frac{\rho \wedge *(\rho \wedge \lambda)}{u} .
$$

If $X \in \operatorname{Vect}(M)$ is a vector field then

$$
*^{\rho} \rho(X, \cdot)=-\rho \wedge g(X, \cdot) .
$$

The map

$$
R^{\rho}: \Omega^{2}(M) \rightarrow \Omega^{2}(M), \quad R^{\rho} \omega:=\omega-\frac{\omega \wedge \rho}{\operatorname{dvol}_{\rho}} \rho
$$

is an involution that preserves the exterior product, acts as the identity on the orthogonal complement of $\rho$ with respect to the exterior product and it sends $\rho$ to $-\rho$. Moreover, it maps $\Omega^{+}$to $\Omega^{+^{\rho}}$, the self-dual forms with respect to the metric $g^{\rho}$. The Hodge star operator $*^{\rho}: \Omega^{2}(M) \rightarrow \Omega^{2}(M)$ associated to $g^{\rho}$ is given by

$$
*^{\rho} \omega=R^{\rho} * R^{\rho} \omega .
$$

Let $\omega \in \Omega^{2}(M)$ be a self-dual two-form and let $J: T M \rightarrow T M$ be an almost complex structure such that $g=\omega(\cdot, J \cdot)$. We define the almost complex structure $J^{\rho}$ by

$$
\rho\left(J^{\rho} \cdot, \cdot\right):=\rho(\cdot, J \cdot)
$$

and a self-dual two-form $\omega^{\rho}$ with respect to $g^{\rho}$ by

$$
\omega^{\rho}:=R^{\rho} \omega
$$

Then,

$$
g^{\rho}=\omega^{\rho}\left(\cdot, J^{\rho} \cdot\right)
$$

and

$$
*^{\rho}\left(\lambda \wedge \omega^{\rho}\right)=\lambda \circ J^{\rho}
$$

for all one-forms $\lambda \in \Omega^{1}(M)$.

Now, fix a symplectic form $\rho \in \mathscr{S}_{a}$. Let $\mathscr{S}_{a+\mathcal{H}^{\rho}}$ be the space of symplectic forms representing a cohomology class in the affine space $a+\mathcal{H}^{\rho} \subset H^{2}(M ; \mathbb{R})$. Define the map $K: \mathscr{S}_{a+\mathcal{H}^{\rho}} \rightarrow \Omega^{+}$by

$$
K(\rho):=\frac{\rho+* \rho}{2 u}, \quad u=\frac{\rho \wedge \rho}{2 \mathrm{dvol}} .
$$

Denote the extension of this map to the Sobolev space $\mathscr{S}_{a+\mathcal{H}_{\rho}}^{k, p}$ also by $K$.

Theorem 1 (The Map K) Let $k-\frac{4}{p}>0$.

(i) For every $\rho \in \mathscr{S}_{a+\mathcal{H}_{\rho}}^{k, p}$, there exists a $W^{k, p}$-neighborhood of $\rho$ such that $K$ restricted to this neighborhood is a diffeomorphism of Banach spaces.

(ii) Let $\mathcal{H} \in H^{2}(M ; \mathbb{R})$ be a positive linear subspace and $a \in H^{2}(M ; \mathbb{R})$. The map $K: \mathscr{S}_{a+\mathcal{H}} \rightarrow \Omega^{+}$is injective. 
(iii) There exist polynomials $\mathfrak{p}_{1}, \mathfrak{p}_{2}$ with positive coefficients with the following significance. If $\rho \in \mathscr{S}^{k, p}$ and $K(\rho) \in W^{k+1, p}\left(M, \Lambda^{+}\right)$with $\frac{1}{u} \leq C<\infty$, then $\rho \in \mathscr{S}^{k+1, p}$ and

$$
\begin{aligned}
\|\rho\|_{W^{k+1, p}} \leq & \mathfrak{p}_{1}\left(C,\|\rho\|_{L^{\infty}}\right)\left\|\frac{\rho^{+}}{u}\right\|_{W^{k+1, p}} \\
& +\mathfrak{p}_{2}\left(C,\|\rho\|_{L^{\infty}},\|\rho\|_{W^{k-1, p}},\left\|\frac{\rho^{+}}{u}\right\|_{W^{k, p}}\right)\|\rho\|_{W^{k, p}} .
\end{aligned}
$$

Proof See page 7.

We will need the following three lemmas to prove Theorem 1.

Lemma 1 (Negative chords) Let $V$ be a real four-dimensional vector space equipped with the standard metric, $\theta \in \Lambda^{+} V$ with $\theta^{2}=\mathrm{dvol}$ and $\rho_{1}, \rho_{2} \in \Lambda^{2} V$ such that

$$
\rho_{i} \wedge \rho_{i}=\mathrm{dvol}, \quad \rho_{i}^{+}=\lambda_{i} \theta, \quad \lambda_{i} \geq 1, \quad i=1,2 .
$$

Then,

$$
\frac{\left(\rho_{1}-\rho_{2}\right)^{2}}{\text { dvol }} \leq 0
$$

and

$$
\frac{\left(\rho_{1}-\rho_{2}\right)^{2}}{\operatorname{dvol}}=0 \Leftrightarrow \rho_{1}=\rho_{2} .
$$

Proof Since $\rho_{1}^{+}=\lambda_{1} \theta$ and $\rho_{2}^{+}=\lambda_{2} \theta$ for $\lambda_{1}, \lambda_{2} \geq 1$, we find

$$
\begin{aligned}
\left(\rho_{1}-\rho_{2}\right)^{2} & =\left(\rho_{1}^{+}-\rho_{2}^{+}+\rho_{1}^{-}-\rho_{2}^{-}\right)^{2} \\
& =\left(\left(\lambda_{1}-\lambda_{2}\right) \theta+\rho_{1}^{-}-\rho_{2}^{-}\right)^{2} \\
& =\left(\lambda_{1}-\lambda_{2}\right)^{2} \mathrm{dvol}+\left(\rho_{1}^{-}-\rho_{2}^{-}\right)^{2} \\
& =\left(\lambda_{1}-\lambda_{2}\right)^{2} \mathrm{dvol}-\left|\rho_{1}^{-}\right|^{2} \mathrm{dvol}-\left|\rho_{2}^{-}\right|^{2} \mathrm{dvol}-2 \rho_{1}^{-} \wedge \rho_{2}^{-} .
\end{aligned}
$$

Thus,

$$
\frac{\left(\rho_{1}-\rho_{2}\right)^{2}}{\operatorname{dvol}}=\left(\lambda_{1}-\lambda_{2}\right)^{2}-\left|\rho_{1}^{-}\right|^{2}-\left|\rho_{2}^{-}\right|^{2}+2\left\langle\rho_{1}^{-}, \rho_{2}^{-}\right\rangle .
$$

Since

$$
\mathrm{dvol}=\rho_{i} \wedge \rho_{i}=\lambda_{i}^{2} \theta \wedge \theta-\rho_{i}^{-} \wedge \rho_{i}^{-}=\left(\lambda_{i}^{2}-\left|\rho_{i}^{-}\right|^{2}\right) \mathrm{dvol}
$$

we have $\left|\rho_{i}^{-}\right|^{2}=\lambda_{i}^{2}-1$ and hence

$$
\begin{aligned}
\frac{\left(\rho_{1}-\rho_{2}\right)^{2}}{\operatorname{dvol}} & =\lambda_{1}^{2}+\lambda_{2}^{2}-2 \lambda_{1} \lambda_{2}-\lambda_{1}^{2}+1-\lambda_{2}^{2}+1+2\left\langle\rho_{1}^{-}, \rho_{2}^{-}\right\rangle \\
& =-2 \lambda_{1} \lambda_{2}+2+2\left\langle\rho_{1}^{-}, \rho_{2}^{-}\right\rangle .
\end{aligned}
$$

By the Cauchy-Schwarz inequality $\left\langle\rho_{1}^{-}, \rho_{2}^{-}\right\rangle \leq\left|\rho_{1}^{-}\right|\left|\rho_{2}^{-}\right|$and equality holds if and only if $\rho_{1}^{-}$and $\rho_{2}^{-}$are colinear. Thus,

$$
\begin{aligned}
\frac{\left(\rho_{1}-\rho_{2}\right)^{2}}{\mathrm{dvol}} & \leq-2 \lambda_{1} \lambda_{2}+2+2\left|\rho_{1}^{-}\right|\left|\rho_{2}^{-}\right| \\
& =-2 \lambda_{1} \lambda_{2}+2+2 \sqrt{\lambda_{1}^{2}-1} \sqrt{\lambda_{2}^{2}-1}
\end{aligned}
$$


and equality holds only if $\rho_{1}^{-}$and $\rho_{2}^{-}$are colinear. Suppose $\left(\rho_{1}-\rho_{2}\right)^{2} \geq 0$. Then,

$$
\lambda_{1} \lambda_{2}-1 \leq \sqrt{\lambda_{1}^{2}-1} \sqrt{\lambda_{2}^{2}-1} .
$$

Squaring both sides of the inequality yields

$$
\lambda_{1}^{2} \lambda_{2}^{2}-2 \lambda_{1} \lambda_{2}+1 \leq\left(\lambda_{1}^{2}-1\right)\left(\lambda_{2}^{2}-1\right)=\lambda_{1}^{2} \lambda_{2}^{2}-\lambda_{1}^{2}-\lambda_{2}^{2}+1 .
$$

Hence, we find

$$
\lambda_{1}^{2}+\lambda_{2}^{2}-2 \lambda_{1} \lambda_{2} \leq 0
$$

and equality holds if and only if $\rho_{1}^{-}$and $\rho_{2}^{-}$are colinear. But clearly

$$
\lambda_{1}^{2}+\lambda_{2}^{2}-2 \lambda_{1} \lambda_{2}=\left(\lambda_{1}-\lambda_{2}\right)^{2} \geq 0
$$

and it follows that $\lambda_{1}=\lambda_{2}$ and $\rho_{1}^{-}$and $\rho_{2}^{-}$are colinear. From $\rho_{i}^{+}=\lambda_{i} \theta$, we get $\rho_{1}^{+}=\rho_{2}^{+}$. Furthermore, since $\lambda_{1}=\lambda_{2}$ and $\left|\rho_{i}^{-}\right|^{2}=\lambda_{i}^{2}-1$, we get $\left|\rho_{1}^{-}\right|=\left|\rho_{2}^{-}\right|$and therefore $\rho_{1}^{-}= \pm \rho_{2}^{-}$. However, if $\rho_{2}=\rho_{1}^{+}-\rho_{1}^{-}$and $\left|\rho_{1}^{-}\right|>0$, then $\left(\rho_{1}-\rho_{2}\right)^{2} \leq 0$ is in violation to our assumption. We conclude that $\rho_{1}=\rho_{2}$.

Remark 1 The set

$$
\mathcal{P}_{\mathrm{dvol}, \theta}:=\left\{\rho \in \Lambda^{2} V \mid \rho \wedge \rho=\mathrm{dvol}, \rho^{+}=\lambda \theta \text { for }|\lambda| \geq 1\right\}
$$

for a fixed volume form dvol and a self-dual two-form $\theta$ was considered by Donaldson in [2] in the context of the following problem: How many symplectic structures $\rho$ exist, such that $\rho \wedge \rho=\mathrm{dvol}$ for a prescribed volume form dvol, $\rho$ is compatible with a prescribed almost complex structure and $\rho$ lays in a given positive affine subspace of $H^{2}(M, \mathbb{R})$ ? The answer is that it is unique. The set $\mathcal{P}_{\mathrm{dvol}, \theta}$ is a three-dimensional submanifold of $\Lambda^{2} V$ with two components. The key property of this manifold established in Lemma 1 is called 'negative chords'.

Lemma 2 (The Linearization of $K$ ) Let $\rho_{s}$ be a path of nondegenerate 2-forms and $\widehat{\rho}=$ $\left.\frac{d}{d s}\right|_{s=0} \rho_{s}, \rho=\rho_{0}$. Then,

$$
\left.\frac{d}{d s}\right|_{s=0}\left(\frac{\rho_{s}^{+}}{u_{s}}\right)=\frac{1}{u} R^{\rho} \widehat{\rho}^{+^{\rho}}, \quad u_{s}=\frac{\rho_{s} \wedge \rho_{s}}{2 \operatorname{dvol}}, \quad R^{\rho} w=w-\frac{w \wedge \rho}{\operatorname{dvol}_{\rho}} \rho .
$$

In particular,

$$
\widehat{\rho}^{+^{\rho}}=\left.u R^{\rho} \frac{d}{d s}\right|_{s=0}\left(\frac{\rho_{s}^{+}}{u_{s}}\right)
$$

Proof We compute

$$
\left.\frac{d}{d s}\right|_{s=0}\left(\frac{\rho_{s}^{+}}{u_{s}}\right)=\frac{\hat{\rho}^{+}}{u}-\frac{\widehat{\rho} \wedge \rho}{\operatorname{dvol}_{\rho}} \frac{\rho^{+}}{u}=\frac{1}{u}\left(R^{\rho} \widehat{\rho}\right)^{+}=\frac{1}{u} R^{\rho} \widehat{\rho}^{+\rho} .
$$

For the last equality, we used that the linear map $R^{\rho}: \Lambda^{2} V \rightarrow \Lambda^{2} V$ is an involution on $\Lambda^{2} V$ for a 4-dimensional real vector space $V$ and it maps $\Lambda^{+}$to $\Lambda^{+^{\rho}}$ and vice versa, where $\Lambda^{+^{\rho}}$ is the space of self-dual 2-forms for the metric $g^{\rho}$ (see [5] for a proof of these facts).

Lemma 3 (Lie Derivative) Let $X$ be a vector field on $M$ and $\rho$ a nondegenerate two-form. Then,

$$
\left(\mathcal{L}_{X} \rho\right)^{+^{\rho}}=u R^{\rho}\left(\mathcal{L}_{X} \frac{\rho^{+}}{u}-\frac{\mathcal{L}_{X} \mathrm{dvol}}{\mathrm{d} \operatorname{dvol}} \frac{\rho^{+}}{u}-\frac{1}{2}\left(\mathcal{L}_{X} *\right) \frac{\rho}{u}\right) .
$$


Proof Let $\psi_{s}, s \geq 0$ be the family of diffeomorphisms on $M$ generated by the vector field $X$. Then,

$$
\begin{aligned}
2 \psi_{s}^{*} \frac{\rho^{+}}{u}= & \psi_{s}^{*}\left((\rho+* \rho) \frac{2 \mathrm{dvol}}{\rho \wedge \rho}\right) \\
= & \left(\psi_{s}^{*} \rho+\left(\psi_{s}^{*} *\right)\left(\psi_{s}^{*} \rho\right)\right) \frac{2 \psi_{s}^{*} \mathrm{dvol}}{\psi_{s}^{*}(\rho \wedge \rho)} \\
= & \left(\psi_{s}^{*} \rho+*\left(\psi_{s}^{*} \rho\right)\right) \frac{2 \mathrm{dvol}}{\psi_{s}^{*}(\rho \wedge \rho)} \frac{2 \psi_{s}^{*} \mathrm{dvol}}{2 \mathrm{dvol}} \\
& +\left(\left(\psi_{s}^{*} *\right)\left(\psi_{s}^{*} \rho\right)-*\left(\psi_{s}^{*} \rho\right)\right) \frac{2 \psi_{s}^{*} \mathrm{dvol}}{\psi_{s}^{*}(\rho \wedge \rho)}
\end{aligned}
$$

Thus,

$$
\begin{aligned}
& \left.\frac{d}{d s}\right|_{s=0}\left(2 \psi_{s}^{*} \frac{\rho^{+}}{u}\right) \\
= & \left.\frac{d}{d s}\right|_{s=0}\left(2\left(\psi_{s}^{*} \rho\right)^{+} \frac{2 \text { dvol }}{\psi_{s}^{*}(\rho \wedge \rho)}\right)+\left.\frac{2 \rho^{+}}{u} \frac{d}{d s}\right|_{s=0} \frac{\psi_{s}^{*} \text { dvol }}{\text { dvol }}+\left.\frac{d}{d s}\right|_{s=0}\left(\psi_{s}^{*} *\right) \frac{\rho}{u} .
\end{aligned}
$$

Using Lemma 2, we then compute

$$
\begin{aligned}
2\left(\mathcal{L}_{X} \rho\right)^{+^{\rho}} & =2\left(\left.\frac{d}{d s}\right|_{s=0}\left(\psi_{s}^{*} \rho\right)\right)^{+^{\rho}} \\
& =\left.u R^{\rho} \frac{d}{d s}\right|_{s=0}\left(2\left(\psi_{s}^{*} \rho\right)^{+} \frac{2 \mathrm{dvol}}{\psi_{s}^{*}(\rho \wedge \rho)}\right) \\
& =u R^{\rho}\left(2 \mathcal{L}_{X} \frac{\rho^{+}}{u}-\frac{2 \rho^{+}}{u} \frac{\mathcal{L}_{X} \mathrm{dvol}}{\operatorname{dvol}}-\left(\mathcal{L}_{X} *\right) \frac{\rho}{u}\right)
\end{aligned}
$$

This proves the lemma.

Proof of Theorem 1 We prove (i). By Lemma 2 the linearization of $K$ is given by

$$
\widehat{K}: T_{\rho} \mathscr{S}_{a+\mathcal{H}_{\rho}}^{k, p} \rightarrow W^{k, p}\left(M, \Lambda^{+}\right): \quad \widehat{\rho} \mapsto \frac{1}{u} R^{\rho} \widehat{\rho}^{+^{\rho}} .
$$

We claim that this is an isomorphism. Then, (i) follows from the inverse function theorem for Banach spaces. By Hodge's theory for the operator $d^{*^{\rho}} d+d d^{*^{\rho}}$ every $\sigma \in \mathscr{S}_{a+\mathcal{H}^{\rho}}^{k, p}$ can be written as a sum $\sigma=\rho+d \lambda+h$ for a unique $\lambda \in W^{k+1, p}\left(M, T^{*} M\right)$ and a $h \in \mathcal{H}^{\rho}$ such that $\int_{M} d \lambda \wedge *^{\rho} h=0$ and $d^{*^{\rho}} \lambda=0$. Hence,

$$
\widehat{\rho}=d \lambda+h
$$

for such unique $\lambda$ and $h$. Now suppose $\widehat{K} \widehat{\rho}=0$. It follows that

$$
d^{\left(+{ }^{\rho}\right)} \lambda+h=\frac{1}{2}\left(d \lambda+*^{\rho} d \lambda\right)+h=0 .
$$

Since $h$ is closed, $d d^{\left(+^{\rho}\right)} \lambda=0$, and thus

$$
0=\int_{M} d d^{+^{\rho}} \lambda \wedge \lambda=-\int_{M} d^{\left(+^{\rho}\right)} \lambda \wedge d \lambda=-\int_{M}\left|d^{\left(+^{\rho}\right)} \lambda\right| \mathrm{dvol} .
$$

Since $0=\int_{M} d \lambda \wedge d \lambda=\int_{M}\left|d^{\left(+^{\rho}\right)} \lambda\right|-\left|d^{\left(-^{\rho}\right)} \lambda\right|$ dvol, it follows that

$$
d \lambda=0 \text {. }
$$


Together with $d^{*^{\rho}} \lambda=0$, this implies that $\lambda$ is harmonic and $h=0$. This shows that $\widehat{K}$ is injective. Now, let $\eta \in W^{k, p}\left(M, \Lambda^{+}\right)$. Since $W^{k, p}$ is closed under products and composition with smooth functions for $k-\frac{4}{p}>0, u_{0} R^{\rho} \eta$ is in $W^{k, p}\left(M, \Lambda^{+^{\rho}}\right)$ and by Hodge's theory there exists a unique one-form $\lambda \in W^{k+1, p}\left(M, T^{*} M\right)$ and a harmonic two-form $h$ which is self-dual with respect to $*^{\rho}$ such that $u_{0} R^{\rho} \eta=d \lambda+h$. Then, $\widehat{K}(d \lambda+h)=\widehat{K}\left(u_{0} R^{\rho} \eta\right)=\eta$ and this shows that $\widehat{K}$ is surjective. This proves (i).

We prove (ii). Let $\mathcal{H} \subset H^{2}(M ; \mathbb{R})$ be a positive linear subspace. Let $\rho_{1}$, $\rho_{2}$ be two elements of $\mathscr{S}_{a+\mathcal{H}}$ such that

$$
\eta:=K\left(\rho_{1}\right)=K\left(\rho_{2}\right)
$$

Define

$$
\theta:=\frac{\eta}{|\eta|^{2}} \in \Omega^{+}
$$

Then,

$$
\theta \wedge \theta=\frac{1}{|\eta|^{2}} \mathrm{dvol}, \quad \rho_{i}^{+}=\lambda_{i} \theta, \quad \lambda_{i}:=|\eta|^{2} u_{i}, \quad u_{i}=\frac{\rho_{i} \wedge \rho_{i}}{2 \mathrm{dvol}}
$$

and

$$
\begin{aligned}
\left(\rho_{i}-\theta\right)^{2} & =\rho_{i} \wedge \rho_{i}-2 \rho_{i}^{+} \wedge \theta+\theta^{2} \\
& =2 u_{i} \mathrm{dvol}-2 \lambda_{i} \theta^{2}+\theta^{2} \\
& =2 u_{i} \mathrm{dvol}-2|\eta|^{2} u_{i} \frac{1}{|\eta|^{2}} \mathrm{dvol}+\theta^{2} \\
& =\theta^{2}
\end{aligned}
$$

for $i=1,2$. It now follows from Lemma 1 that

$$
\left(\rho_{1}-\rho_{2}\right)^{2}=\left(\left(\rho_{1}-\theta\right)-\left(\rho_{2}-\theta\right)\right)^{2} \leq 0
$$

pointwise. On the other hand, since $\left[\rho_{1}\right],\left[\rho_{2}\right] \in a+\mathcal{H}$ and $\mathcal{H}$ is a positive subspace of $H^{2}(M)$, we have

$$
\int_{M}\left(\rho_{1}-\rho_{2}\right)^{2} \geq 0
$$

and therefore $\left(\rho_{1}-\rho_{2}\right)^{2}=0$. We conclude with Lemma 1 that $\rho_{1}=\rho_{2}$. This proves (ii).

We prove (iii). Let $\rho \in \mathscr{S}^{k, p}$ and let $\mathcal{L} \rho$ be a Lie derivative of $\rho$ in an arbitrary direction. By Cartan's formula the Lie derivative of $\rho$ is exact and by Lemma 3

$$
\begin{aligned}
\left(d+d^{*^{\rho}}\right) \mathcal{L} \rho & =d^{*^{\rho}} \mathcal{L} \rho \\
& =-*^{\rho} d *^{\rho} \mathcal{L} \rho \\
& =-*^{\rho} d\left((\mathcal{L} \rho)^{+^{\rho}}-(\mathcal{L} \rho)^{-^{\rho}}\right) \\
& =-2 *^{\rho} d(\mathcal{L} \rho)^{+^{\rho}} \\
& =-*^{\rho} d\left(u R^{\rho}\left(\mathcal{L} \frac{\rho^{+}}{u}-\frac{\mathcal{L} \mathrm{dvol}}{\operatorname{dvol}} \frac{\rho^{+}}{u}-\frac{1}{2} \frac{(\mathcal{L} *) \rho}{u}\right)\right) .
\end{aligned}
$$

The right hand side is a term of the form

$$
P_{1}\left(\frac{1}{u}, \rho\right) \partial^{2} \frac{\rho^{+}}{u}+P_{2}\left(\frac{1}{u}, \rho\right) \partial \rho \partial \frac{\rho^{+}}{u}+P_{3}\left(\frac{1}{u}, \rho\right) \partial \rho
$$


for polynomials $P_{1}, P_{2}, P_{3}$ with smooth coefficient functions in the indicated variables. It follows from elliptic regularity theory and product estimates for Sobolev spaces (see, e.g. [6, Lemma A.2]) that $\mathcal{L} \rho \in W^{k, p}$ and that there exist polynomials $\mathfrak{p}_{1}, \mathfrak{p}_{2}$ with positive coefficients independent of $\rho$ with the following significance

$$
\begin{aligned}
\|\mathcal{L} \rho\|_{W^{k, p}} \leq & \mathfrak{p}_{1}\left(C,\|\rho\|_{L^{\infty}}\right)\left\|\frac{\rho^{+}}{u}\right\|_{W^{k+1, p}} \\
& +\mathfrak{p}_{2}\left(C,\|\rho\|_{L^{\infty}},\|\rho\|_{W^{k-1, p}},\left\|\frac{\rho^{+}}{u}\right\|_{W^{k, p}}\right)\|\rho\|_{W^{k, p}}
\end{aligned}
$$

for $C=\sup _{x \in M} \frac{1}{u(x)}$. Since the Lie derivative $\mathcal{L} \rho$ was arbitrary, the result follows.

\section{The Evolution Equation for $K(\rho)$}

In view of Theorem 1, the Donaldson flow has an equivalent description on the space of selfdual two-forms given by the evolution equation for $K(\rho)=\frac{\rho^{+}}{u}$. This equation exposes the parabolic nature of the Donaldson flow equation and it is the key for the regularity theorems which we will prove in the later sections.

To obtain a global formula, we introduce the operator $S^{\rho}$,

$$
S^{\rho}: \Omega^{1} \rightarrow \Omega^{+}, \quad S^{\rho} \lambda:=-R^{\rho} d^{+^{\rho}} \lambda+u \nabla_{X_{\lambda}} \frac{\rho^{+}}{u},
$$

where $\lambda \in \Omega^{1}, R^{\rho}$ is defined by (4) and $\rho\left(X_{\lambda}, \cdot\right):=\lambda$. We say $\omega_{1}, \omega_{2}, \omega_{3} \in \Omega^{+}$form a standard local frame of $\Omega^{+}$if and only if locally

$$
\omega_{i} \wedge \omega_{j}= \begin{cases}2 \mathrm{dvol}, & i=j \\ 0, & i \neq j\end{cases}
$$

Theorem 2 (The Evolution of $\frac{\rho^{+}}{u}$ )

(i) Suppose $\rho$ is a smooth solution to the Donaldson flow equation. Then, the evolution of the 2-form $\frac{\rho^{+}}{u}$ is given by the equation

$$
\partial_{t} \frac{\rho^{+}}{u}=\frac{1}{u} R^{\rho} d^{+^{\rho}} *^{\rho} d \theta^{\rho}, \quad u=\frac{\rho \wedge \rho}{2 \mathrm{dvol}}, \quad \theta^{\rho}=\frac{2 \rho^{+}}{u}-\left|\frac{\rho^{+}}{u}\right| \rho .
$$

Here, $R^{\rho}$ is defined by (4).

(ii) (8) is the same as

$$
\partial_{t} \frac{\rho^{+}}{u}=-\frac{2}{u} S^{\rho}\left(S^{\rho}\right)^{*^{\rho}} \frac{\rho^{+}}{u}+\nabla_{X_{* \rho} d \theta^{\rho}} \frac{\rho^{+}}{u},
$$

where $S^{\rho}$ is defined by (7), $\left(S^{\rho}\right)^{*^{\rho}}$ is the adjoint of $S^{\rho}$ with respect to the inner products $\int_{M}\langle\cdot, \cdot\rangle_{g} \rho \mathrm{dvol}$ on $\Omega^{1}$ and $\int_{M}\langle\cdot, \cdot\rangle_{g} \mathrm{dvol}$ on $\Omega^{+}$and

$$
\rho\left(X_{* \rho} d \theta^{\rho}, \cdot\right):=*^{\rho} d \theta^{\rho} .
$$


(iii) Let $\omega_{1}, \omega_{2}, \omega_{3}$ form a local standard frame of $\Omega^{+}$. Then, the evolution of the functions $K_{i}:=\frac{\rho \wedge \omega_{i}}{\mathrm{dvol}_{\rho}}$ is given by

$$
\begin{aligned}
& \partial_{t} \sum_{i} K_{i} \omega_{i} \\
= & -\sum_{i, j, k \text { cyclic }}\left(\frac{1}{u} d^{*^{\rho}} d K_{i}-2\left\{K_{j}, K_{k}\right\}_{\rho}-\rho\left(X_{K_{i}}, \sum_{\ell} J_{\ell} X_{K_{\ell}}\right)\right) \omega_{i} \\
& -\frac{1}{u} E_{\omega}^{\rho} K+E_{\omega}^{\prime \rho} K .
\end{aligned}
$$

Here, $X_{H}$ denotes the Hamiltonian vector field of the function $H$. The bracket $\{\cdot, \cdot\}_{\rho}$ denotes the Poisson bracket with respect to the symplectic structure $\rho . E_{\omega}^{\rho}$ and $E_{\omega}^{\prime \rho}$ are error terms depending on the frame $\omega_{1}, \omega_{2}, \omega_{3}$ that vanish whenever $\nabla \omega_{i}=0$ and are given by

$$
\begin{aligned}
E_{w}^{\rho} f:= & \sum_{i, j}\left\langle\left(S^{\rho}\right)^{*^{\rho}} \omega_{i}, d f_{j} \circ J_{j}^{\rho}\right\rangle_{\rho} \omega_{i}+2 S^{\rho} \sum_{j} f_{j}\left(S^{\rho}\right)^{*^{\rho}} \omega_{j} \\
& +\sum_{i, j, k \text { cyclic }} *^{\rho}\left(d f_{j} \wedge d \omega_{k}-d f_{k} \wedge d \omega_{j}\right) \omega_{i} \\
E_{\omega}^{\prime \rho} f:= & \sum_{i, j} K_{j} d f_{i}\left(X_{\left(S^{\rho}\right)^{* \rho} \omega_{j}}\right) \omega_{i}+\sum_{i} f_{i} \nabla_{X_{* \rho} d \theta^{\rho}} \omega_{i}
\end{aligned}
$$

for $f=\left(f_{1}, f_{2}, f_{3}\right) \in \Omega^{0}\left(M, \mathbb{R}^{3}\right)$.

(iv) Assume the hyperKähler case. Then, the evolution of the functions $K_{i}=\frac{\rho \wedge \omega_{i}}{\mathrm{dvol}_{\rho}}$ is given by

$$
\partial_{t} \sum_{i} K_{i} \omega_{i}=-\sum_{i, j, k \text { cyclic }}\left(\frac{1}{u} d^{*^{\rho}} d K_{i}-2\left\{K_{j}, K_{k}\right\}_{\rho}-\rho\left(X_{K_{i}}, \sum_{\ell} J_{\ell} X_{K_{\ell}}\right)\right) \omega_{i} .
$$

Proof See page 13 .

We need the following lemma on the properties of $S^{\rho}$ and its adjoint $\left(S^{\rho}\right)^{*^{\rho}}$.

Lemma 4 (The Operators $\left.S^{\rho},\left(S^{\rho}\right)^{*^{\rho}}\right)$ Let $\rho \in \mathscr{S}_{a}$.

(i) The adjoint of $S^{\rho}: \Omega^{1}(M) \rightarrow \Omega^{+}(M)$ with respect to the inner products $\int_{M}\langle\cdot, \cdot\rangle_{g} \rho \mathrm{dvol}$ on $\Omega^{1}$ and $\int_{M}\langle\cdot, \cdot\rangle_{g} \mathrm{dvol}$ on $\Omega^{+}$is given by

$$
\left(S^{\rho}\right)^{*^{\rho}} \xi:=-d^{*^{\rho}}\left(R^{\rho} \xi\right)+*^{\rho}\left(g\left(\nabla \frac{\rho^{+}}{u}, \xi\right) \wedge \rho\right),
$$

where $g\left(\nabla \frac{\rho^{+}}{u}, \xi\right)$ is the 1-form given by $X \mapsto g\left(\nabla_{X} \frac{\rho^{+}}{u}, \xi\right)$ for a vector field $X$.

(ii) $\left(S^{\rho}\right)^{*^{\rho}} \frac{2 \rho^{+}}{u}=*^{\rho} d \theta^{\rho}$, where $\theta^{\rho}=\frac{2 \rho^{+}}{u}+\left|\frac{\rho^{+}}{u}\right|^{2} \rho$.

(iii) Let $\omega \in \Omega^{+}$. Then,

$$
\left(S^{\rho}\right)^{*^{\rho}} \omega=*^{\rho}\left(d \omega-g\left(\nabla \omega, \frac{\rho^{+}}{u}\right) \wedge \rho\right) .
$$

In particular, $\left(S^{\rho}\right)^{*^{\rho}} \omega$ is independent of derivatives of $\rho$ and if $\nabla \omega=0$, then $\left(S^{\rho}\right)^{*^{\rho}} \omega=0$. 
(iv) The operator $\left(S^{\rho}\right)^{*^{\rho}}$ satisfies the following Leibniz rule,

$$
\left(S^{\rho}\right)^{*^{\rho}}(f \xi)=*^{\rho}\left(d f \wedge R^{\rho} \xi\right)+f\left(S^{\rho}\right)^{*^{\rho}} \xi
$$

for all $\xi \in \Omega^{+}$and $f \in \Omega^{0}(M, \mathbb{R})$.

(v) Let $\omega_{1}, \omega_{2}, \omega_{3}$ be a standard frame for $\Omega^{+}$with respect to the background metric $g$ and $f_{i} \in \Omega^{0}(M, \mathbb{R})$, then

$$
\left(S^{\rho}\right)^{*^{\rho}}\left(\sum_{i} f_{i} \omega_{i}\right)=\sum_{i}\left(d f_{i} \circ J_{i}^{\rho}+f_{i}\left(S^{\rho}\right)^{*^{\rho}} \omega_{i}\right) .
$$

(vi) In the hyperKähler case,

$$
\left(S^{\rho}\right)^{*^{\rho}}\left(\sum_{i} f_{i} \omega_{i}\right)=\sum_{i} d f_{i} \circ J_{i}^{\rho} .
$$

(vii) Let $\omega_{1}, \omega_{2}, \omega_{3}$ be a standard frame for $\Omega^{+}$with respect to the background metric $g$ and $f_{i} \in \Omega^{0}(M, \mathbb{R})$, then

$$
2 S^{\rho}\left(S^{\rho}\right)^{*^{\rho}}\left(\sum_{j} f_{j} \omega_{j}\right)=\sum_{i, j, k \text { cyclic }}\left(d^{*^{\rho}} d f_{i}-u\left(\left\{f_{j}, K_{k}^{\rho}\right\}_{\rho}-\left\{K_{j}^{\rho}, f_{k}\right\}_{\rho}\right)\right) \omega_{i}+E_{w}^{\rho} f,
$$

where $K_{i}^{\rho}:=\frac{\omega_{i} \wedge \rho}{\operatorname{dvol}_{\rho}}$ and $E_{\omega}^{\rho}: \Omega^{0}\left(M, \mathbb{R}^{3}\right) \rightarrow \Omega^{+}$is the linear first order differential operator given by (9). It vanishes whenever $\nabla \omega_{i}=0$ for $i=1,2,3$.

Proof We prove (i). To compute the adjoint of $S^{\rho}$ let $\lambda$ be a 1 -form and $\xi \in \Omega^{+}$, then

$$
\begin{aligned}
& \int_{M}\left\langle-d^{*^{\rho}}\left(R^{\rho} \xi\right)+*^{\rho}\left(g\left(\nabla \frac{\rho^{+}}{u}, \xi\right) \wedge \rho\right), \lambda\right\rangle_{g^{\rho}} \mathrm{dvol} \\
= & \int_{M}\left\langle\xi,-R^{\rho} d^{+^{\rho}} \lambda\right\rangle_{g} \mathrm{dvol}+\int_{M}\left\langle\rho\left(Y_{g\left(\nabla \frac{\rho^{+}}{u}, \xi\right)}, \cdot\right), \rho\left(X_{\lambda}, \cdot\right)\right\rangle_{g \rho} \mathrm{dvol},
\end{aligned}
$$

where

$$
g\left(Y_{g\left(\nabla \frac{\rho^{+}}{u}, \xi\right)}, \cdot\right):=g\left(\nabla \frac{\rho^{+}}{u}, \xi\right), \quad \rho\left(X_{\lambda}, \cdot\right):=\lambda .
$$

Here, we used the identity

$$
*^{\rho} \iota(X) \rho=-\rho \wedge g(X, \cdot)
$$

in the last equation. Using this identity again together with the definition of the Hodge star operator $*^{\rho}$, we have

$$
\begin{aligned}
\int_{M}\left\langle\rho\left(Y_{g\left(\nabla \frac{\rho^{+}}{u}, \xi\right)}, \cdot\right), \rho\left(X_{\lambda}, \cdot\right)\right\rangle_{g \rho} \mathrm{dvol} & =\int_{M} g\left(Y_{g\left(\nabla \frac{\rho^{+}}{u}, \xi\right)}, X_{\lambda}\right) \mathrm{dvol}_{\rho} \\
& =\int_{M} g\left(\nabla_{X_{\lambda}} \frac{\rho^{+}}{u}, \xi\right) \mathrm{dvol}_{\rho} .
\end{aligned}
$$

Thus, we have proved that

$$
\begin{aligned}
& \int_{M}\left\langle-d^{*^{\rho}}\left(R^{\rho} \xi\right)+*^{\rho}\left(g\left(\nabla \frac{\rho^{+}}{u}, \xi\right) \wedge \rho\right), \lambda\right\rangle_{g^{\rho}} \mathrm{dvol} \\
= & \int_{M}\left\langle\xi,-R^{\rho} d^{+^{\rho}} \lambda+u \nabla_{X_{\lambda}} \frac{\rho^{+}}{u}\right\rangle_{g} \mathrm{dvol}
\end{aligned}
$$


for all self-dual two-forms $\xi \in \Omega^{+}(M)$ and one-forms $\lambda \in \Omega^{1}(M)$. This proves (i).

Part (ii) follows from the computation

$$
\begin{aligned}
\left(S^{\rho}\right)^{*^{\rho}} \frac{2 \rho^{+}}{u} & =*^{\rho} d R^{\rho} \frac{2 \rho^{+}}{u}+*^{\rho}\left(2 g\left(\nabla \frac{\rho^{+}}{u}, \frac{\rho^{+}}{u}\right) \wedge \rho\right) \\
& =*^{\rho} d\left(R^{\rho} \frac{2 \rho^{+}}{u}+\left|\frac{\rho^{+}}{u}\right|^{2} \rho\right) \\
& =*^{\rho} d\left(\frac{2 \rho^{+}}{u}-\frac{\rho \wedge \frac{2 \rho^{+}}{u}}{\operatorname{dvol}_{\rho}} \rho+\left|\frac{\rho^{+}}{u}\right|^{2} \rho\right) \\
& =*^{\rho} d\left(\frac{2 \rho^{+}}{u}-\left|\frac{\rho^{+}}{u}\right|^{2} \rho\right) \\
& =*^{\rho} d \theta^{\rho},
\end{aligned}
$$

where we used that $*^{\rho} R^{\rho} \frac{2 \rho^{+}}{u}=R^{\rho} \frac{2 \rho^{+}}{u}$ in the first equation. This proves (ii).

We prove (iii). Let $\omega \in \Omega^{+}$. Observe that

$$
\begin{aligned}
d\left(R^{\rho} \omega\right) & =d\left(\omega-\frac{\omega \wedge \rho}{\operatorname{dvol}_{\rho}} \rho\right) \\
& =d \omega-\left(g\left(\nabla \omega, \frac{\rho^{+}}{u}\right)+g\left(\omega, \nabla \frac{\rho^{+}}{u}\right)\right) \wedge \rho
\end{aligned}
$$

and hence

$$
\begin{aligned}
\left(S^{\rho}\right)^{*^{\rho}} \omega & =*^{\rho} d\left(R^{\rho} \omega\right)+*^{\rho}\left(g\left(\nabla \frac{\rho^{+}}{u}, \omega\right) \wedge \rho\right) \\
& =*^{\rho}\left(d \omega-\left(g\left(\nabla \omega, \frac{\rho^{+}}{u}\right) \wedge \rho\right)\right) .
\end{aligned}
$$

Now suppose $\nabla \omega=0$. Then, we have $d \omega=0$ and it follows from the last equation that $\left(S^{\rho}\right)^{*^{\rho}} \omega=0$. This proves (iii).

We prove (iv). Let $\xi \in \Omega^{+}$and $f \in \Omega^{0}(M, \mathbb{R})$. Then,

$$
\begin{aligned}
\left(S^{\rho}\right)^{*^{\rho}}(f \xi) & =*^{\rho} d\left(R^{\rho} f \xi\right)+*^{\rho}\left(g\left(\nabla \frac{\rho^{+}}{u}, f \xi\right) \wedge \rho\right) \\
& =*^{\rho}\left(d f \wedge R^{\rho} \xi\right)+f *^{\rho} d\left(R^{\rho} \xi\right)+f *^{\rho}\left(g\left(\nabla \frac{\rho^{+}}{u}, \xi\right) \wedge \rho\right) \\
& =*^{\rho}\left(d f \wedge R^{\rho} \xi\right)+f\left(S^{\rho}\right)^{*^{\rho}} \xi .
\end{aligned}
$$

This proves (iv).

We prove (v). It follows from (iv) that

$$
\begin{aligned}
\left(S^{\rho}\right)^{*^{\rho}}\left(\sum_{i} f_{i} \omega_{i}\right) & =\sum_{i}\left(*^{\rho}\left(d f_{i} \wedge R^{\rho} \omega_{i}\right)+f_{i}\left(S^{\rho}\right)^{*^{\rho}} \omega_{i}\right) \\
& =\sum_{i}\left(d f_{i} \circ J_{i}^{\rho}+f_{i}\left(S^{\rho}\right)^{*^{\rho}} \omega_{i}\right),
\end{aligned}
$$

where the last equality follows from identity (5). This proves (v).

(vi) follows directly from (v) since $\left(S^{\rho}\right)^{*^{\rho}} \omega_{i}=0$ by (iii) for the hyperKähler structures $\omega_{1}, \omega_{2}, \omega_{3}$. 
We prove (vii). It follows from (v) that in a standard frame $\omega_{1}, \omega_{2}, \omega_{3}$ for $\Omega^{+}, S^{\rho}$ is given by

$$
S^{\rho} \lambda=\frac{1}{2} \sum_{i}\left(-d^{*^{\rho}}\left(\lambda \circ J_{i}^{\rho}\right)+\left\langle\left(S^{\rho}\right)^{*^{\rho}} \omega_{i}, \lambda\right\rangle_{\rho}\right) \omega_{i}
$$

for a 1 -form $\lambda$. Again by (v)

$$
\begin{aligned}
2 S^{\rho}\left(S^{\rho}\right)^{*^{\rho}} \sum_{j} f_{j} \omega_{j}= & 2 S^{\rho}\left(\sum_{j} d f_{j} \circ J_{j}^{\rho}+f_{j}\left(S^{\rho}\right)^{*^{\rho}} \omega_{j}\right) \\
= & \sum_{i, j}-d^{*^{\rho}}\left(d f_{j} \circ J_{j}^{\rho} \circ J_{i}^{\rho}\right) \omega_{i} \\
& +\sum_{i}\left\langle\left(S^{\rho}\right)^{*^{\rho}} \omega_{i}, \sum_{j} d f_{j} \circ J_{j}^{\rho}\right\rangle_{\rho} \omega_{i}+2 S^{\rho} \sum_{j} f_{j}\left(S^{\rho}\right)^{*^{\rho}} \omega_{j} .
\end{aligned}
$$

Observe that

$$
d\left(R^{\rho} \omega_{i}\right)=d\left(\omega_{i}-K_{i}^{\rho} \rho\right)=d \omega_{i}-d K_{i}^{\rho} \wedge \rho .
$$

Then by identity (5) and the hyperKähler relations for $J_{i}^{\rho}$,

$$
\begin{aligned}
& d *^{\rho}\left(\sum_{j} d f_{j} \circ J_{j}^{\rho} \circ J_{1}^{\rho}\right)=d *^{\rho}\left(-d f_{1}-d f_{2} \circ J_{3}^{\rho}+d f_{3} \circ J_{2}^{\rho}\right) \\
= & -d *^{\rho} d f_{1}+d\left(d f_{2} \wedge R^{\rho} \omega_{3}-d f_{3} \wedge R^{\rho} \omega_{2}\right) \\
= & -d *^{\rho} d f_{1}-d f_{2} \wedge d K_{3}^{\rho} \wedge \rho+d f_{3} \wedge d K_{2}^{\rho} \wedge \rho+d f_{2} \wedge d \omega_{3}-d f_{3} \wedge d \omega_{2} \\
= & -d *^{\rho} d f_{1}-\iota\left(X_{f_{2}}\right) \rho \wedge \iota\left(X_{K_{3}^{\rho}}\right) \rho \wedge \rho+\iota\left(X_{f_{3}}\right) \rho \wedge \iota\left(X_{K_{2}^{\rho}}\right) \rho \wedge \rho \\
& +d f_{2} \wedge d \omega_{3}-d f_{3} \wedge d \omega_{2} \\
= & -d *^{\rho} d f_{1}-\left\{f_{2}, K_{3}^{\rho}\right\}_{\rho} \operatorname{dvol}_{\rho}-\left\{K_{2}^{\rho}, f_{3}\right\}_{\rho} \operatorname{dvol}_{\rho}+d f_{2} \wedge d \omega_{3}-d f_{3} \wedge d \omega_{2}
\end{aligned}
$$

and hence

$$
\begin{aligned}
\sum_{i, j}-d^{*^{\rho}}\left(d f_{j} \circ J_{j}^{\rho} \circ J_{i}^{\rho}\right) \omega_{i}= & \sum_{i, j, k \text { cyclic }}\left(d^{*^{\rho}} d f_{i}-u\left(\left\{f_{j}, K_{k}^{\rho}\right\}_{\rho}-\left\{K_{j}^{\rho}, f_{k}\right\}_{\rho}\right)\right) \omega_{i} \\
& +\sum_{i, j, k \text { cyclic }} *^{\rho}\left(d f_{j} \wedge d \omega_{k}-d f_{k} \wedge d \omega_{j}\right) \omega_{i} .
\end{aligned}
$$

This proves (vii).

We end the section with a proof of Theorem 2 .

Proof of Theorem 2 We prove (i). Let $\rho$ be a smooth solution to the Donaldson flow equation $\partial_{t} \rho=d *^{\rho} d \theta^{\rho}$. Then by (6),

$$
\partial_{t} \frac{\rho^{+}}{u}=\frac{1}{u}\left(R^{\rho} \partial_{t} \rho\right)^{+}=\frac{1}{u} R^{\rho}\left(\partial_{t} \rho\right)^{+^{\rho}}=\frac{1}{u} R^{\rho} d^{+^{\rho}} *^{\rho} d \theta^{\rho} .
$$

Here, we use that $R^{\rho}$ maps $\Lambda^{+}$to $\Lambda^{+^{\rho}}$ and vise versa in the second equation. This proves (i).

We prove (ii). By the definition of the operator $S^{\rho}$ given by (7) and Lemma 4 (ii),

$$
-\frac{2}{u} S^{\rho}\left(S^{\rho}\right)^{*^{\rho}} \frac{\rho^{+}}{u}=-\frac{1}{u} S^{\rho}\left(*^{\rho} d \theta^{\rho}\right)=\frac{1}{u} R^{\rho} d^{+^{\rho}} *^{\rho} d \theta^{\rho}-\nabla_{X_{*} \rho d \theta^{\rho}} \frac{\rho^{+}}{u} .
$$


Together with (i), this proves (ii).

We prove (iii). First note that in a local standard frame $\omega_{1}, \omega_{2}, \omega_{3}$ for $\Omega^{+}$we have $\frac{2 \rho^{+}}{u}=\sum_{i} K_{i} \omega_{i}$ and by Lemma 4 (ii) and (v)

$$
*^{\rho} d \theta^{\rho}=\left(S^{\rho}\right)^{*^{\rho}} \frac{2 \rho^{+}}{u}=\sum_{i}\left(d K_{i} \circ J_{i}^{\rho}+K_{i}\left(S^{\rho}\right)^{*^{\rho}} \omega_{i}\right) .
$$

Since

$$
\rho\left(J_{i} X_{K_{i}}, \cdot\right)=\rho\left(X_{K_{i}}, J_{i}^{\rho} \cdot\right)
$$

the vector field $X_{* \rho} d \theta^{\rho}$ that satisfies $\rho\left(X_{* \rho} d \theta^{\rho}, \cdot\right)=*^{\rho} d \theta^{\rho}$ is given by

$$
\sum_{j}\left(J_{j} X_{K_{j}}+K_{j} X_{\left(S^{\rho}\right)^{* \rho} \omega_{j}}\right) \text {. }
$$

Hence,

$$
\begin{aligned}
\nabla_{X_{* \rho} \rho} \frac{2 \rho^{+}}{u}= & \nabla_{X_{*} \rho d \theta} \sum_{i} K_{i} \omega_{i} \\
= & \sum_{i}\left(d K_{i}\left(\sum_{j} J_{j} X_{K_{j}}\right)+\sum_{j} K_{j} d K_{i}\left(X_{\left(S^{\rho}\right)^{* \rho} \omega_{j}}\right)\right) \omega_{i} \\
& +\sum_{i} K_{i} \nabla_{X_{* \rho} \rho \theta^{\rho}} \omega_{i} \\
= & \sum_{i} \rho\left(X_{K_{i}}, \sum_{j} J_{j} X_{K_{i}}\right) \omega_{i}+\sum_{i, j} K_{j} d K_{i}\left(X_{\left(S^{\rho}\right)^{* \rho} \omega_{j}}\right) \omega_{i} \\
& +\sum_{i} K_{i} \nabla_{X_{*} \rho d \theta \rho} \omega_{i} \\
= & \sum_{i} \rho\left(X_{K_{i}}, \sum_{j} J_{j} X_{K_{i}}\right) \omega_{i}+E_{\omega}^{\prime \rho} K
\end{aligned}
$$

for $K=\left(K_{1}, K_{2}, K_{3}\right)$. By Lemma 4 (vii)

$$
\begin{aligned}
\frac{2}{u} S^{\rho}\left(S^{\rho}\right)^{*^{\rho}} \frac{2 \rho^{+}}{u} & =\frac{2}{u} S^{\rho}\left(S^{\rho}\right)^{*^{\rho}}\left(\sum_{j} K_{j} \omega_{j}\right) \\
& =\sum_{i, j, k \text { cyclic }}\left(\frac{1}{u} d^{*^{\rho}} d K_{i}-2\left\{K_{j}, K_{k}\right\}_{\rho}\right) \omega_{i}+\frac{1}{u} E_{\omega}^{\rho} K
\end{aligned}
$$

Thus, from

$$
\partial_{t} \frac{2 \rho^{+}}{u}=-\frac{2}{u}\left(S^{\rho}\right)^{*^{\rho}} S^{\rho} \frac{2 \rho^{+}}{u}+\nabla_{X_{*} \rho d \theta} \frac{2 \rho^{+}}{u}
$$

we find that

$$
\begin{aligned}
\partial_{t} \sum_{i} K_{i} \omega_{i}= & -\sum_{i, j, k \text { cyclic }}\left(\frac{1}{u} d^{*^{\rho}} d K_{i}-2\left\{K_{j}, K_{k}\right\}_{\rho}-\rho\left(X_{K_{i}}, \sum_{\ell} J_{\ell} X_{K_{\ell}}\right)\right) \omega_{i} \\
& -\frac{1}{u} E_{\omega}^{\rho} K+E_{\omega}^{\prime \rho}(K) .
\end{aligned}
$$


We prove (iv). Since $\nabla \omega_{i}=0$ for the three hyperKähler structures $\omega_{1}, \omega_{2}, \omega_{3}$ we have $d \omega_{i}=0$ and $\left(S^{\rho}\right)^{*^{\rho}} \omega_{i}=0$. Hence, the error terms $E_{\omega}^{\rho}$ and $E^{\prime}{ }_{\omega}$ vanish and (iv) follows from (iii).

\section{Regularity}

In this section, we prove that a solution to the Donaldson flow equation is as smooth as its initial condition allows, given that it is an element of $L^{2}\left(I, W^{2, p}\right) \cap W^{1,2}\left(I, L^{p}\right)$ for $p>4$. In particular, it is smooth if its initial conditions are smooth. The proof combines two insights. First, the regularity of $\frac{\rho^{+}}{u}$ determines the regularity of $\rho$. This is the content of Theorem 1 (iii). Second, the evolution of $\frac{\rho^{+}}{u}$ is given by a parabolic operator, where the right hand side of the equation is essentially a product of two derivatives. This is the content of Theorem 2 (iii). This allows bootstrapping. The details are given in the next two theorems. The first theorem illustrates the ideas in the simpler case of a critical point.

Theorem 3 (Critical Point) Let $p>4$ and let $\rho \in W^{1, p}\left(M, \Lambda^{2}\right)$ be a critical point of the Donaldson flow. Then, $\rho$ is smooth.

The next lemma will be needed in the bootstrapping process.

Lemma 5 Let $p^{\prime}>p>4$. If $\rho \in W^{1, p}$ is a symplectic form with $\frac{\rho^{+}}{u} \in W^{1, p^{\prime}}$ then $\rho \in W^{1, p^{\prime}}$ as well.

Proof Let $\mathcal{L} \rho$ be a Lie derivative of $\rho$ in an arbitrary direction. Let $\rho_{0}$ be a smooth nondegenerate form such that $\left\|\rho-\rho_{0}\right\|_{L^{\infty}}<\delta$ for a small $\delta>0$. Then, there exists a unique 1 -form $\lambda \in W^{1, p}$ such that $d \lambda=\mathcal{L} \rho$ and $d^{*^{\rho_{0}}} \lambda=0$. By elliptic regularity theory for the operator $d^{+^{\rho_{0}}}+d^{*^{\rho_{0}}}$ there exists a constant $c>0$ such that

$$
\|\lambda\|_{W^{1, p^{\prime}}} \leq c\left(\left\|(d \lambda)^{+^{\rho_{0}}}\right\|_{L^{p^{\prime}}}+\|d \lambda\|_{L^{2}}\right) .
$$

Since

$$
\left\|\left(*^{\rho}-*^{\rho_{0}}\right) d \lambda\right\|_{L^{p^{\prime}}} \leq \mathfrak{p}\left(\left\|\rho-\rho_{0}\right\|_{L^{\infty}}\right)\|d \lambda\|_{L^{p^{\prime}}}
$$

for a polynomial $\mathfrak{p}$ with positive coefficients vanishing at zero, we have

$$
\left\|(d \lambda)^{+^{\rho_{0}}}\right\|_{L^{p^{\prime}}} \leq\left\|(d \lambda)^{+^{\rho}}\right\|_{L^{p^{\prime}}}+\delta\|d \lambda\|_{L^{p^{\prime}}}
$$

for a small enough $\delta>0$. Further, since

$$
0=\int_{M} d \lambda \wedge d \lambda=\int_{M}\left|d \lambda^{+^{\rho}}\right|^{2}-\left|d \lambda^{-^{\rho}}\right|^{2} \mathrm{dvol}
$$

we have

$$
\|d \lambda\|_{L^{2}} \leq 2\left\|(d \lambda)^{+^{\rho}}\right\|_{L^{2}} .
$$

By Lemma 3,

$$
(\mathcal{L} \rho)^{+^{\rho}}=u R^{\rho}\left(\mathcal{L} \frac{\rho^{+}}{u}-\frac{\mathcal{L} \mathrm{dvol}}{\operatorname{dvol}} \frac{\rho^{+}}{u}-\frac{1}{2} \frac{(\mathcal{L} *) \rho}{u}\right)
$$


Therefore,

$$
\begin{aligned}
\|\lambda\|_{W^{1, p^{\prime}}} & \leq c\left(\left\|(\mathcal{L} \lambda)^{+^{\rho}}\right\|_{L^{p^{\prime}}}+\delta\|d \lambda\|_{L^{p^{\prime}}}+2\left\|(d \lambda)^{+^{\rho}}\right\|_{L^{2}}\right) \\
& \leq \mathfrak{p}_{1}\left(C,\|\rho\|_{L^{\infty}}\right)\left\|\mathcal{L} \frac{\rho^{+}}{u}\right\|_{L^{p^{\prime}}}+\mathfrak{p}_{2}\left(C,\|\rho\|_{L^{\infty}}\right)+c \delta\|\lambda\|_{W^{1, p^{\prime}}}
\end{aligned}
$$

for polynomials with positive coefficient $\mathfrak{p}_{1}$ and $\mathfrak{p}_{2}$, and

$$
C:=\sup _{x \in M} \frac{1}{u(x)}, \quad u:=\frac{\rho \wedge \rho}{2 \mathrm{dvol}} .
$$

Since this is true for an arbitrary Lie derivative of $\rho$ and small enough $\delta>0$, it follows that

$$
\rho \in W^{1, p^{\prime}} \text {. }
$$

This proves the lemma.

We will need the following lemma on elliptic regularity of the operator $d^{*^{\rho}} \frac{d}{u}$ : $C^{\infty}(M) \rightarrow C^{\infty}(M)$ in the case that $\rho$ and thus the coefficients are not smooth.

Lemma 6 (Elliptic Regularity) Let $p>4, q>1, k \geq 0$ and let $\rho \in W^{k+1, p}\left(M, \Lambda^{2}\right)$ such that $\rho \wedge \rho>0$. Assume there exists a constant $c_{0}>0$ such that for all $v, w \in C^{\infty}(M)$ and all $\varepsilon>0$ we can estimate

$$
\|\partial v \partial w\|_{W^{k, q}} \leq c_{0}\|v\|_{W^{k+1, p}}\left(\frac{1}{\varepsilon}\|w\|_{L^{q}}+\varepsilon\|w\|_{W^{k+2, q}}\right) .
$$

Let $v \in W^{1, q}(M)$ and $f \in W^{k, q}(M)$ such that

$$
\int_{M} \frac{1}{u} d v \wedge *^{\rho} d \phi=\int_{M} f \varphi \mathrm{dvol}
$$

for all $\varphi \in C^{1}(M)$. Then, $v$ is an element of $W^{k+2, q}(M)$ and there exists a constant $c=$ $c\left(q, k, M,\|\rho\|_{W^{1, p}}\right)$ such that

$$
\|v\|_{W^{k+2, q}} \leq c\left(\|f\|_{W^{k, q}}+\|v\|_{L^{q}}\right) .
$$

Proof We only prove the case $k=0$, the general case follows by induction over $k$. Choose coordinate charts for $M$ and a subordinate partition of unity of $M$. Let $\psi \in C_{0}^{\infty}(M)$ be a cutoff function. Then, we have

$$
\int_{M} \frac{1}{u} d(\psi v) \wedge *^{\rho} d \varphi=\int_{M} f^{\prime} \varphi \mathrm{dvol}
$$

for all $\varphi \in C^{1}(M)$ and

$$
f^{\prime}:=\psi f-\left(d^{*^{\rho}} \frac{d}{u} \psi\right) v+*^{\rho}\left(d v \wedge *^{\rho} \frac{d}{u} \psi\right) .
$$

Let $B \subset \mathbb{R}^{4}$ be a ball around zero. Let $\triangle$ be the Hodge laplacian on $\mathbb{R}^{4}$ with respect to the standard metric. We know from ellpitic regularity if $v \in W_{0}^{1, p}(B)$ is a weak solution to the equation

$$
\triangle v=f
$$

in the sense that for every $\varphi \in C_{0}^{1}(B)$ we have

$$
\int_{B} d v \wedge * d \varphi=\int_{B} f \varphi \mathrm{dvol}
$$


for an $f \in L^{q}(B)$ and $q>1$, then $v \in W^{2, q}(B)$ and there exists a constant $c_{1}(q, B)$ such that

$$
\|v\|_{W^{2, q}(B)} \leq c_{1}\left(\|f\|_{L^{q}(B)}+\|v\|_{L^{q}(B)}\right) .
$$

We choose a coordinate chart such that the image of the support of $\psi$ is contained in $B$ and the push-forward of $\rho$ equals the standard symplectic structure at $0 \in B$. We can always achieve this by a change of coordinates. Let us denote the push-forward of $\rho$ under this coordinate by $\rho_{\alpha}$. Furthermore, we denote by $\triangle^{\rho_{\alpha}}$ the operator $d^{*^{\rho}} \frac{d}{u}$ expressed in this chart and by $v_{\alpha}, f_{\alpha}^{\prime}$ the push-forward of $\psi v, f^{\prime}$, respectively. By estimate (10), there exists a polynomial with positive coefficents $\mathfrak{p}$ such that

$$
\begin{aligned}
& \quad\left\|\left(\triangle^{\rho_{\alpha}}-\triangle\right) v_{\alpha}\right\|_{L^{q}} \\
& \leq \mathfrak{p}\left(\left\|\rho_{\alpha}\right\|_{L^{\infty}}\right)\left(\left\|\rho_{\alpha}-\rho(0)\right\|_{L^{\infty}}\left\|v_{\alpha}\right\|_{W^{2, q}}+\left\|\partial \rho_{\alpha} \partial v_{\alpha}\right\|_{L^{q}}\right) \\
& \leq \mathfrak{p}\left(\left\|\rho_{\alpha}\right\|_{L^{\infty}}\right)\left(\left\|\rho_{\alpha}-\rho(0)\right\|_{L^{\infty}}\left\|v_{\alpha}\right\|_{W^{2, q}}\right. \\
& \left.\quad+\left\|\rho_{\alpha}\right\|_{W^{1, p}}\left(\frac{1}{\varepsilon}\left\|v_{\alpha}\right\|_{L^{q}}+\varepsilon\left\|v_{\alpha}\right\|_{W^{2, q}}\right)\right) .
\end{aligned}
$$

By interpolating $\left\|\partial v_{\alpha}\right\|_{L^{q}} \leq c\left\|v_{\alpha}\right\|_{L^{q}}^{\frac{1}{2}}\left\|v_{\alpha}\right\|_{W^{2, q}}^{\frac{1}{2}}$ with the Galgliardo-Nirenberg interpolation inequality, we find

$$
\left\|f_{\alpha}^{\prime}\right\|_{L^{q}} \leq c_{2}\|f\|_{L^{q}}+c_{3}\left(\frac{1}{\varepsilon}\left\|v_{\alpha}\right\|_{L^{q}}+\varepsilon \|\left. v_{\alpha}\right|_{W^{2, q}}\right) .
$$

If $v_{\alpha}$ is a smooth solution to

$$
\triangle^{\rho_{\alpha}} v_{\alpha}=f_{\alpha}^{\prime}
$$

then it also solves

$$
\triangle v_{\alpha}=f_{\alpha}^{\prime}+\left(\triangle-\Delta^{\rho_{\alpha}}\right) v_{\alpha}
$$

By choosing $\varepsilon$ and the ball $B$ small, we see that there exits a constant $c_{4}=c_{4}\left(q,\|\rho\|_{W^{1, p}}\right)$ such that

$$
\|v\|_{W^{2, q}} \leq c_{4}\left(\|f\|_{L^{q}}+\|v\|_{L^{q}}\right) .
$$

Here, we are using that $W^{1, p}(M) \hookrightarrow C^{0}(M)$ for $p>4$ and therefore $\left\|\rho_{\alpha}-\rho_{0}\right\|_{L^{\infty}(B)}$ is small for a small ball. If $v \in W^{1, q}(M)$ is a weak solution to $d^{*^{\rho}} \frac{d}{u} v=f$ in the sense that

$$
\int_{M} \frac{1}{u} d v \wedge *^{\rho} d \varphi=\int_{M} f \varphi \mathrm{dvol}
$$

for all $\varphi \in C^{1}(M)$ then we approximate $f$ and $\rho$ by smooth functions $f_{k}$, respectively, smooth nondegenerate two-forms $\rho_{k}$, such that

$$
\lim _{k \rightarrow \infty}\left\|f_{k}-f\right\|_{L^{q}}=0, \quad \lim _{k \rightarrow \infty}\left\|\rho-\rho_{k}\right\|_{W^{1, p}}=0 .
$$

For each pair $\left(f_{k}, \rho_{k}\right)$ we find by standard $L^{2}$-theory for elliptic operators a smooth function $v_{k}$ that solves

$$
d^{*^{\rho_{k}}} \frac{d}{u_{k}} v_{k}=f_{k},
$$

and $\int_{M} v_{k} \mathrm{dvol}=\int_{M} v$ dvol. The constant $c_{4}$ in (12) can be choosen uniformly in $k$ for big $k$ and it follows that $\left\{v_{k}\right\}_{k \in \mathbb{N}}$ has a weakly convergent subsequence with limit $\bar{v} \in W^{2, q}$ that satisfies the estimate (12) and $d^{*^{\rho}} \frac{d}{u} \bar{v}=f$. Hence,

$$
\int_{M} \frac{1}{u} d(v-\bar{v}) \wedge *^{\rho} d \varphi=0
$$


for all $\varphi \in C^{1}(M)$. By choosing a sequence $\varphi_{k} \in C^{1}(M)$ such that $\varphi_{k}$ converges to $(v-\bar{v})$ in $W^{1,2}(M)$ we see that $\bar{v}=v$. This proves the lemma.

Proof of Theorem 3 If $\rho$ is a critical point of the Donaldson flow then it follows from Theorem 2 (iii) that for a local standard frame $\omega_{1}, \omega_{2}, \omega_{3}$ and

$$
K_{i}=\frac{\rho \wedge \omega_{i}}{\operatorname{dvol}_{\rho}}
$$

we have the equations

$$
-\frac{1}{u} d^{*^{\rho}} d K_{i}=-2\left\{K_{j}, K_{k}\right\}_{\rho}-\rho\left(X_{K_{i}}, \sum_{\ell} J_{\ell} X_{K_{\ell}}\right)+\frac{\left(\frac{1}{u} E_{\omega}^{\rho}-E_{\omega}^{\prime \rho}\right) \wedge \omega \omega_{i}}{2 \mathrm{dvol}}
$$

for cyclic permutations of $i, j, k$. The right hand side of this equation consists of products of derivatives of the functions $K_{i}$ times a polynomial in the $\rho$ and $\frac{1}{u}$ variables plus lower order terms in the functions $K_{i}$ times another polynomial of the same form. Thus, schematically we may write

$$
d^{*^{\rho}} d K=P_{1}\left(\frac{1}{u}, \rho\right) \partial K \cdot \partial K+P_{2}\left(\frac{1}{u}, \rho\right) \partial K .
$$

Since $1-\frac{4}{p}>0, \rho \in C^{0}$ and since we assume that $\rho$ is a symplectic structure, we have $\sup _{M} \frac{1}{u}<\infty$. It follows that the $L^{\infty}$-norms of $P_{1}, P_{2}$ are bounded. Using Hölder's inequality we see that the right hand side is an element of $L^{\frac{p}{2}}$. For two functions $v, w \in C^{\infty}(M)$, we have the estimate

$$
\|\partial v \partial w\|_{L^{\frac{p}{2}}} \leq\|\partial v\|_{L^{p}}\|\partial w\|_{L^{p}} \leq\|v\|_{W^{1, p}}\left(\frac{1}{\varepsilon}\|w\|_{L^{p}}+\varepsilon\|w\|_{W^{2, p}}\right),
$$

where we used Hölder's inequality in the first inequality and the Gagliardo-Nirenberg interpolation inequality in the second. It follows from Lemma 6 that $K$ is in $W^{2, \frac{p}{2}}$. By Rellich's embedding theorem $W^{1, \frac{p}{2}} \hookrightarrow L^{p^{\prime}}$ where

$$
p^{\prime}=\frac{4 p}{8-p} \text {. }
$$

For $4<p<8$, we have

$$
p^{\prime}>p .
$$

Thus, $K \in W^{1, p^{\prime}}$ and by Lemma $5 \rho \in W^{1, p^{\prime}}$. If we repeat this argument with $p$ replaced by $p^{\prime}$, we find that $K \in W^{1, \frac{p^{\prime \prime}}{2}}, p^{\prime \prime}>p^{\prime}$ and

$$
p^{\prime \prime}-p^{\prime}>p^{\prime}-p \text {. }
$$

Hence, eventually we find that $K \in W^{2, q}$ and $\rho \in W^{1, q}$ for all $q \geq p$. Now, we can use Theorem 1 to see that $\rho \in W^{2, q}$ as well. This implies that the right hand side of (13) is in $W^{1, q}$ and elliptic regularity gives us that $K \in W^{3, q}$. Now, an obvious iteration of these arguments using elliptic regularity and Theorem 1 deduces the regularity of $\rho$ from the regularity of $K$, proving the theorem.

Let $T>0$ and

$$
I:=[0, T) \subset \mathbb{R}
$$


For $p>4$, and integers $k \geq 2,0 \leq r \leq\left\lfloor\frac{k}{2}\right\rfloor$ define the Sobolev space $W^{r, k, p}$ of functions on $I \times M$ such that the weak derivatives $\partial_{t}^{s} \partial^{\ell}$ exist and are bounded in the $L^{p}-L^{2}$-norm for $2 s+\ell \leq k, s \leq r$,

$W^{r, k, p}\left(M_{I}\right):=L^{2}\left(I, W^{k, p}(M)\right) \cap W^{1,2}\left(I, W^{k-2, p}(M)\right) \cap \cdots \cap W^{r, 2}\left(I, W^{k-2 r, p}(M)\right)$.

This definition extends in an obvious way to functions from $I$ to the space of sections of a vector bundle over $M$. In the case at hand, the relevant vector bundle is the bundle of two-forms over $M$ and the corresponding space will be denoted by

$$
W^{r, k, p}\left(M_{I}, \Lambda^{2}\right) \text {. }
$$

If the vector bundle in question is clear, we will simply write $W^{r, k, p}$. The norm on this space is given by

$$
\|u\|_{W^{r, k, p}}:=\sum_{\substack{2 s+\ell \leq k \\ s \leq r}}\left(\int_{M}\left(\left\|\partial_{t}^{s} u\right\|_{W^{\ell, p}}\right)^{2} d t\right)^{\frac{1}{2}} .
$$

The Besov space $B_{q}^{s, p}\left(\mathbb{R}^{n}, \mathbb{C}\right)$ (see [4]) is the completion of the space $C_{0}^{\infty}\left(\mathbb{R}^{n}, \mathbb{C}\right.$ ) with respect to the norm

$$
\|f\|_{B_{q}^{s, p}}:=\|f\|_{L^{p}}+\|f\|_{b_{q}^{s, p}}
$$

for $f \in C_{0}^{\infty}\left(\mathbb{R}^{n}, \mathbb{C}\right)$, where

$$
\|f\|_{b_{q}^{s, p}}^{q}:=\int_{0}^{\infty} \frac{\sup _{|h| \leq r}\left(\int_{\mathbb{R}^{n}}|f(x+h)-2 f(x)+f(x-h)|^{p} d x\right)^{q / p}}{r^{s q}} \frac{d r}{r} .
$$

An excellent self-contained exposition of parabolic $L^{p}-L^{q}$ estimates and the role of Besov spaces for the initial conditions of parabolic flows can be found in [8]. The next proposition shows that the Besov space $B_{2}^{k-1, p}\left(M, \Lambda^{2}\right)$ is the natural space for the initial conditions for solutions $u \in W^{r, k, p}\left(M_{I}, \Lambda^{2}\right)$ of a parabolic evolution equation.

Proposition 1 (Besov Spaces) Let $k \geq 2$.

1) The restriction map $\rho \mapsto \rho(t=0, \cdot)$ extends to a bounded linear operator

$$
W^{r, k, p}\left(M_{I}, \Lambda^{2}\right) \rightarrow B_{2}^{k-1, p}\left(M, \Lambda^{2}\right),
$$

where $B_{2}^{k-1, p}\left(M, \Lambda^{2}\right)$ denotes the Besov space with exponents $p$ and $q=2$.

2) This restriction map is surjective and it has a bounded right inverse.

3) The identity operator on the space of real smooth functions on I to sections in the vector bundle $\Lambda^{2}$ over $M$ with compact support extends to a bounded linear operator

$$
W^{r, k, p}\left(M_{I}, \Lambda^{2}\right) \rightarrow C^{0}\left(I, B_{2}^{k-1, p}\left(M, \Lambda^{2}\right)\right) .
$$

4) Let $k \geq 1$. It holds

$$
W^{k, 2}\left(M, \Lambda^{2}\right)=B_{2}^{k, 2}\left(M, \Lambda^{2}\right)
$$

Proof For $k=2$, the statements 1), 2) and 3) follow directly from [8, Corollary 1.7 and Corollary 1.8] after choosing local coordinates for $M$ and a subordinate partition of unity. For $k=1$, 4) follows from [8, Corollary 13.9 iii)]. 1)-4) can be extended to higher $k$ by using [8, Theorem 14.1 iii)]. 
Theorem 4 (Flow Lines) Let $\rho \in W^{1,2}\left(I, L^{p}\right) \cap L^{2}\left(I, W^{2, p}\right)$ be a solution to the Donaldson flow equation for $p>4$ with initial condition $\rho(t=0, \cdot)=\rho_{0}$. For every integer $k \geq 1$ and all $4<p^{\prime}<p$ the following are equivalent:

1) $\rho_{0} \in B_{2}^{k, p^{\prime}}\left(M, \Lambda^{2}\right)$.

2) $\rho \in W^{\frac{k+1}{2}, k+1, p^{\prime}}\left(M, \Lambda^{2}\right)$.

The proof of this theorem uses parabolic regularity theory. In particular, we use the 'maximal regularity' property of parabolic operators in divergence form. We refer to [7] for these results. The maximal regularity property is usually formulated for operators with time independent smooth coefficients in divergence form, the Hodge laplacian being the archetypal example. The next lemma assures that the operator

$$
d^{*^{\rho}} \frac{d}{u}: C^{\infty}(M) \rightarrow C^{\infty}(M)
$$

has the maximal regularity property as well, even though its coefficients depend on time and are non-smooth in our used case.

Lemma 7 (Maximal Regularity) Let $p>4, q>1$. Let

$$
\rho \in W^{1,2}\left(\mathbb{R}, L^{p}\left(M, \Lambda^{2}\right)\right) \cap L^{2}\left(\mathbb{R}, W^{2, p}\left(M, \Lambda^{2}\right)\right)
$$

be a path of nondegenerate forms. Assume that there exists a constant $c_{0}>0$ such that for all $v, w \in C^{\infty}(M)$ and all $\varepsilon>0$ we can estimate

$$
\|\partial v \partial w\|_{L^{q}} \leq c_{0}\|v\|_{W^{1, p}}\left(\varepsilon\|w\|_{W^{2, q}}+\frac{1}{\varepsilon}\|w\|_{L^{q}}\right) .
$$

Then for all $v \in C_{0}^{\infty}\left(\mathbb{R}, C_{0}^{\infty}(M)\right)$, there exists a constant $c\left(q,\|\rho\|_{L^{\infty}\left(\mathbb{R}, W^{1, p}\right)}\right)>0$ such that

$$
\left\|\partial_{t} v\right\|_{L^{2}\left(\mathbb{R}, L^{q}\right)} \leq c\left\|\partial_{t} v+d^{*^{\rho}} \frac{d}{u} v\right\|_{L^{2}\left(\mathbb{R}, L^{q}\right)}+\|v\|_{L^{2}\left(\mathbb{R}, L^{q}\right)} .
$$

Proof Choose coordinate charts for $\mathbb{R} \times M$ and a subordinate partition of unity. Let $\psi \in C^{\infty}(\mathbb{R} \times M)$ be a cutoff function. Let $I \times B \in \mathbb{R} \times \mathbb{R}^{4}$ be a ball around $(0,0)$. Choose a coordinate chart such that the support of $\psi$ is mapped into $I \times B$ and such that the pushforward of $\rho$ under this coordinate chart at $(0,0)$ equals the standard symplectic structure on $\mathbb{R}^{4}$. We can always achieve this by a change of coordinates. Let us denote the pushforward of $\rho$ under this coordinate chart by $\rho_{\alpha}$ and the operator $d^{*^{\rho}} \frac{d}{u}$ expressed in this chart by $\triangle^{\rho_{\alpha}}$. Further we denote by $v_{\alpha}$ the function $\psi v$ expressed in this chart. From maximal regularity for the standard Laplace operator $\Delta$ on $\mathbb{R} \times \mathbb{R}^{4}$ there exists a constant $c_{1}=c_{1}(q)>0$ such that

$$
\begin{aligned}
\left\|\partial_{t} v_{\alpha}\right\|_{L^{2}\left(\mathbb{R}, L^{q}\right)} & \leq c_{1}\left\|\partial_{t} v_{\alpha}+\Delta v_{\alpha}\right\|_{L^{2}\left(\mathbb{R}, L^{q}\right)} \\
& \leq c_{1}\left\|\partial_{t} v_{\alpha}+\triangle^{\rho_{\alpha}} v_{\alpha}\right\|_{L^{2}\left(\mathbb{R}, L^{q}\right)}+\left\|\left(\Delta-\triangle^{\rho_{\alpha}}\right) v_{\alpha}\right\|_{L^{2}\left(\mathbb{R}, L^{q}\right)} .
\end{aligned}
$$

By choosing the partition of unity such that $\psi$ has small enough support and using the estimate (11), we find that there exists a constant

$$
c_{2}=c_{2}\left(q,\|\rho\|_{L^{\infty}\left(\mathbb{R}, W^{1, p}\right)}\right)
$$

such that

$$
\left\|\partial_{t} v_{\alpha}\right\|_{L^{2}\left(\mathbb{R}, L^{q}\right)} \leq c_{2}\left\|\partial_{t} v_{\alpha}+\triangle^{\rho_{\alpha}} v_{\alpha}\right\|_{L^{2}\left(\mathbb{R}, L^{q}\right)}+\left\|v_{\alpha}\right\|_{L^{2}\left(\mathbb{R}, L^{q}\right)}
$$


From this the global estimate,

$$
\left\|\partial_{t} v\right\|_{L^{2}\left(\mathbb{R}, L^{q}\right)} \leq c_{3}\left\|\partial_{t} v+d^{*^{\rho}} \frac{d}{u} v\right\|_{L^{2}\left(\mathbb{R}, L^{2}\right)}+\|v\|_{L^{2}\left(\mathbb{R}, L^{q}\right)}
$$

follows by choosing a partition of unity such that the previous estimates hold for all chart domains and by estimating additional first order terms appearing from the multiplication of $v$ with the cutoff functions with the Gagliardo-Nirenberg interpolation inequality. This proves the lemma.

Proof of Theorem 4 Let $\rho(t=0, \cdot)=\rho_{0} \in B_{2}^{2, p}$ and let

$$
\widetilde{\rho}_{0} \in W^{1,2}\left(I, W^{1, p}\right) \cap L^{2}\left(I, W^{3, p}\right)
$$

be an extension of $\rho_{0}$ with $\widetilde{\rho}_{0}(t=0, \cdot)=\rho_{0}$. From Theorem 2 (iii), it follows that the evolution of the functions $K_{i}=\frac{\rho \wedge \omega_{i}}{\operatorname{dvol}_{\rho}}$ in a local standard frame $\omega_{1}, \omega_{2}, \omega_{3}$ is given by

$$
\partial_{t} K_{i}+\frac{1}{u} d^{*^{\rho}} d K_{i}=P_{1}\left(\frac{1}{u}, \rho\right) \partial K \cdot \partial K+P_{2}\left(\frac{1}{u}, \rho\right) \partial K,
$$

where $K=K_{k}$ for an arbitrary $k \in\{1,2,3\}, \partial$ is an arbitrary space derivative and $P_{1,2}\left(\frac{1}{u}, \rho\right)$ are polynomials in the variables $\frac{1}{u}$ and $\rho$ with coefficients that are smooth functions on the manifold. Let

$$
\widetilde{K}_{i, 0}:=\frac{\omega_{i} \wedge \widetilde{\rho}_{0}}{\operatorname{dvol} \widetilde{\rho}_{0}}, \quad \widehat{K}_{i}:=K_{i}-\widetilde{K}_{i, 0} .
$$

The evolution of the functions $\widehat{K}_{i}$ is given by the equations

$$
\begin{aligned}
\partial_{t} \widehat{K}_{i}+d^{*^{\rho}} \frac{d}{u} \widehat{K}_{i} & =P_{3}\left(\frac{1}{u}, \rho\right) \partial \rho \cdot \partial K+P_{2}\left(\frac{1}{u}, \rho\right) \partial K-\left(\partial_{t}+\frac{1}{u} d^{*^{\rho}} d\right) \widetilde{K}_{0, i} \\
\widehat{K}_{i}(t=0, \cdot) & =0
\end{aligned}
$$

for a polynom $P_{3}$ with smooth coefficient functions. By assumption, $\rho$ is an element of $W^{1,2}\left(I, L^{p}\right) \cap L^{2}\left(I, W^{2, p}\right)$ and therefore $\rho, K \in C^{0}\left(I, W^{1, p}\right)$. It follows from Hölder's inequality that

$$
\partial \rho \cdot \partial K \in L^{2}\left(I, W^{1, \frac{p}{2}}\right)
$$

Since $W^{1, p} \subset C^{0}$ for $p>4$, we have

$$
\left\|P_{2,3}\right\|_{L^{\infty}\left(I, L^{\infty}\right)}<\infty .
$$

The last term on the right handside of $(15)$ is in $L^{2}\left(I, W^{1, p}\right)$, hence the right hand side of (15) is in $L^{2}\left(I, W^{1, \frac{p}{2}}\right)$. As in the critical point case, the estimate (14) is valid for any two functions $v, w \in C^{\infty}(M)$. Then by the ellipitic regularity Lemma 6 and the maximal regularity Lemma 7, we have

$$
\widehat{K} \in W^{1,2}\left(I, W^{1, \frac{p}{2}}\right) \cap L^{2}\left(I, W^{3, \frac{p}{2}}\right) .
$$

By Rellich's theorem,

$$
\widehat{K} \in C^{0}\left(I, W^{1, p^{\prime}}\right), \quad p^{\prime}=\frac{4 p}{8-p} .
$$

For $4<p<8$ we have

$$
p^{\prime}>p
$$


Since $\widetilde{K}_{0} \in C^{0}\left(I, W^{2, p}\right) \subseteq C^{0}\left(I, C^{1}\right)$, it follows that $K \in C^{0}\left(I, W^{1, p^{\prime}}\right)$. Then with Lemma 5 , we find

$$
\rho \in C^{0}\left(I, W^{1, p^{\prime}}\right) .
$$

If we now repeat these arguments with $p$ replaced by $p^{\prime}$, we find that $\widehat{K} \in C^{0}\left(I, W^{1, p^{\prime \prime}}\right)$ with $p^{\prime \prime}>p^{\prime}$ and

$$
p^{\prime \prime}-p^{\prime}>p^{\prime}-p
$$

Hence, we eventually find that

$$
\widehat{K}, K \in C^{0}\left(I, W^{1, q}\right)
$$

for all $q \geq 1$. In particular, the right hand side of $(15)$ is in $L^{2}\left(I, W^{1, p^{\prime \prime \prime}}\right)$ for a $4<p^{\prime \prime \prime}<p$ and

$$
K \in W^{1,2}\left(I, W^{1, p^{\prime \prime \prime}}\right) \cap L^{2}\left(I, W^{3, p^{\prime \prime \prime}}\right) .
$$

We claim that the following implications hold for $k \geq 3, p>4$,

$$
\begin{gathered}
K \in W^{\left\lfloor\frac{k}{2}\right\rfloor, k, p}, \rho \in W^{\left\lfloor\frac{k-1}{2}\right\rfloor, k-1, p} \Rightarrow \rho \in W^{\left\lfloor\frac{k}{2}\right\rfloor, k, p} \\
\rho, K \in W^{\left\lfloor\frac{k}{2}\right\rfloor, k, p} \Rightarrow P_{1}\left(\frac{1}{u}, \rho\right) \partial K \cdot \partial K+P_{2}\left(\frac{1}{u}, \rho\right) \partial K \in W^{\left\lfloor\frac{k-1}{2}\right\rfloor, k-1, p} \\
P_{1}\left(\frac{1}{u}, \rho\right) \partial K \cdot \partial K+P_{2}\left(\frac{1}{u}, \rho\right) \partial K \in W^{\left\lfloor\frac{k-1}{2}\right\rfloor, k-1, p}, \rho \in W^{\left\lfloor\frac{k}{2}\right\rfloor, k, p}, \rho_{0} \in B_{2}^{k, p} \\
\Rightarrow K \in W^{\left\lfloor\frac{k+1}{2}\right\rfloor, k+1, p} .
\end{gathered}
$$

The statement of the theorem then follows by induction over $k \geq 3$ and $p=p^{\prime \prime \prime}$. We prove the first implication. It follows from Theorem 1 that $\rho \in L^{2}\left(I, W^{k, p}\right)$ under the stated assertions. Then from the Donaldson flow equation,

$$
\left\|\partial_{t} \rho\right\|_{W^{\left\lfloor\frac{k}{2}\right\rfloor-1, k-2, p}}=\left\|d *^{\rho} d \theta^{\rho}\right\|_{W^{\left\lfloor\frac{k}{2}\right\rfloor-1, k-2, p}} .
$$

Because the expressions $*^{\rho}, \theta^{\rho}$ are given by polynomials in the variables $\rho, \frac{1}{u}$, this expression is bounded in terms of a polynomial with positive real coefficients of the Sobolev norms of its factors (see, e.g. [6, A.2 and A.3]). The second implication follows again using the product estimates in Sobolev spaces. To see the third implication, note that there exists an extension of $\rho_{0}$ to an element $\widetilde{\rho}_{0}$ such that $\widetilde{K}_{0} \in W^{\left\lfloor\frac{k+1}{2}\right\rfloor, k+1, p}$. It follows from (15) and maximal regularity for the operator $\frac{1}{u} d^{*^{\rho}} d$ that $\widehat{K} \in W^{\left\lfloor\frac{k+1}{2}\right\rfloor, k+1, p}$ and hence so is $K$. This proves the theorem.

The following is an immediate corollary to Theorem 4 .

Corollary 1 (Instant Regularity) Let $\rho \in L^{2}\left(I, W^{2, p}\right) \cap W^{1,2}\left(I, L^{p}\right)$ be a solution to the Donaldson flow equation. For all $4<p^{\prime}<p$ the following holds true. If $\rho(t=0, \cdot) \in$ $B_{2}^{k, p^{\prime}}$ then the map $t \mapsto \rho(t, \cdot)$ is a continous map from $(0, T)$ to $B_{2}^{k+1, p^{\prime}}$. In particular, $\rho(t, \cdot)$ is smooth for all $t \in(0, T)$.

Remark 2 Theorem 4 can be refined by using a more sophisticated machinery of maximal $L^{p}-L^{q}$-regularity and the theory of Besov spaces. The correct space for the initial condition for a solution in the space

$$
L^{q}\left(I, W^{k, p}\right) \cap W^{1, q}\left(I, W^{k-2, p}\right) \cap \ldots \cap W^{k, q}\left(I, L^{p}\right)
$$


is the Besov space $B_{q}^{s, p}$ for

$$
s:=k-\frac{2}{q} .
$$

For $s p>4$ a solution is continuous in space and time and this bound is sharp. The argument of Theorem 4 can be used to show that a solution in this space but initial conditions in $B_{q}^{s+1, p}$ is in

$$
L^{q}\left(I, W^{k+1, p}\right) \cap W^{1, q}\left(I, W^{k-1, p}\right) \cap \ldots \cap W^{k, q}\left(I, W^{1, p}\right) .
$$

Acknowledgements The author would like to thank Dietmar Salamon for many insightful discussions.

Funding Open Access funding provided by ETH Zurich. This research was partially supported by the Swiss National Science Foundation Grant 200021-127136 and performed when the author was a Ph.D. student at ETH Zürich.

Open Access This article is licensed under a Creative Commons Attribution 4.0 International License, which permits use, sharing, adaptation, distribution and reproduction in any medium or format, as long as you give appropriate credit to the original author(s) and the source, provide a link to the Creative Commons licence, and indicate if changes were made. The images or other third party material in this article are included in the article's Creative Commons licence, unless indicated otherwise in a credit line to the material. If material is not included in the article's Creative Commons licence and your intended use is not permitted by statutory regulation or exceeds the permitted use, you will need to obtain permission directly from the copyright holder. To view a copy of this licence, visit http://creativecommons.org/licenses/by/4.0/.

\section{References}

1. Donaldson, S.K.: Moment maps and diffeomorphisms. Asian J. Math. 3, 1-16 (1999)

2. Donaldson, S.K.: Two-forms on four-manifolds and Elliptic equations. Nankai Tracts in Mathematics. World Scientific 11, 153-172 (2006)

3. Friedman, A.: Partial Differential Equations. Robert E. Krieger Publishing Company, Huntington (1976)

4. Grigor'yan, A., Liu, L.: Heat kernel and Lipschitz-Besov spaces. Forum Math. 27(6), 3567-3613 (2015)

5. Krom, R.S., Salamon, D.A.: The Donaldson geometric flow for symplectic four-manifolds. J. Symplectic Geom. 17, 381-417 (2019)

6. Krom, R.S.: The Donaldson geometric flow is a local smooth semiflow. arXiv:1512.09199 (2015)

7. Lamberton, L.: Équations d'évolution linéaires associées à des semi-groupes de contraction dans les espace $L^{p}$. J. Funct. Anal. 72(2), 252-262 (1987)

8. Salamon, D.A.: Parabolic $L^{p}-L^{q}$ estimates. ETH Preprint. http://www.math.ethz.ch/ ${ }^{\text {salamon/ }}$ PREPRINTS/parabolic.pdf (2016)

9. Salamon, D.A.: Uniqueness of symplectic structures. Acta Math. Vietnam. 38, 123-144 (2013)

Publisher's Note Springer Nature remains neutral with regard to jurisdictional claims in published maps and institutional affiliations. 